\title{
Locally Acquired mcr-1 in Escherichia coli, Australia, 2011 and 2013
}

\author{
Justin A. Ellem, Andrew N. Ginn, \\ Sharon C.-A. Chen, John Ferguson, \\ Sally R. Partridge, Jonathan R. Iredell
}

We identified discrete importation events of the mcr-1 gene on incompatibility group Incl2 plasmids in Escherichia coli isolated from patients in New South Wales, Australia, in 2011 and 2013. mcr-1 is present in a small minority of colistin-resistant Enterobacteriaceae and appears not to be established locally.

$\mathrm{T}$ he $m c r-1$ (mobile colistin resistance) gene was discovered in Escherichia coli isolates collected during 20112014 from animals and meat products and from Klebsiella pneumoniae from human patients in China (1). Since then, $m c r-1$ has been identified on plasmids of various incompatibility (Inc) types associated with 0,1 , or 2 copies of the insertion sequence (IS) ISApl1 (2), and it has been identified in other species (most notably Salmonella spp.) and in several other countries (3), not including Australia. We analyzed colistin resistance in Enterobacteriaceae isolates collected in Sydney, New South Wales, Australia, during 2007-2016.

\section{The Study}

We reviewed 4,555 isolates of the family Enterobacteriaceae from 2007-2016 that were available for further testing for colistin (polymyxin E) resistance, excluding species that are intrinsically resistant. These isolates were from specimens tested at or referred to the Centre for Infectious Diseases Microbiology Laboratory Services at Westmead Hospital, Sydney, New South Wales, Australia, and were all resistant to third-generation cephalosporins, carbapenems,

Author affiliations: Westmead Hospital, Westmead, New South Wales, Australia (J.A. Ellem, A.N. Ginn, S.C.-A. Chen, S.R. Partridge, J.R Iredell); New South Wales Health Pathology, Westmead (J.A. Ellem, A.N. Ginn, S.C.-A. Chen, J.R. Iredell); The University of Sydney, Sydney, New South Wales, Australia (A.N. Ginn, S.C.-A. Chen, S.R. Partridge, J.R. Iredell); The Westmead Institute for Medical Research, Westmead (A.N. Ginn, S.R. Partridge, J.R. Iredell); John Hunter Hospital, Newcastle, New South Wales, Australia (J. Ferguson); Pathology North, Newcastle (J. Ferguson); University of Newcastle, Newcastle (J. Ferguson)

DOI: https://dx.doi.org/10.3201/eid2307.161638 or both. For antimicrobial drug susceptibility testing, we used the Phoenix Automated Microbiology System (panels NMIC/ID-80, NMIC/ID-101, and NMIC-203; Becton Dickinson, Franklin Lakes, NJ, USA). Of the 4,555 isolates, $96(2.1 \%)$ had a colistin (polymyxin E) minimum inhibitory concentration (MIC) of $\geq 2 \mu \mathrm{g} / \mathrm{mL}$, which corresponds to EUCAST (European Committee on Antimicrobial Susceptibility Testing) breakpoints (http://www. eucast.org/clinical_breakpoints/). The 96 colistin-resistant isolates consisted of $44 \mathrm{~K}$. pneumoniae, $8 \mathrm{~K}$. oxytoca, 18 E. coli, 19 Enterobacter spp., 5 Hafnia alvei, and 2 Citrobacter freundii isolates. By using published primers (1), we identified $m c r-1$ in 2 of the colistin-resistant $E$. coli isolates; these isolates were from patients in different cities in New South Wales.

In September 2011, E. coli JIE2288 (colistin MIC of $4 \mu \mathrm{g} / \mathrm{mL}$ ) was isolated from the urine of a 70-year-old woman after she had been in the intensive care unit (ICU) at Westmead Hospital, a large metropolitan hospital, for 2 months for management of a subarachnoid hemorrhage. The woman was not administered colistin/polymyxin antimicrobial drugs during hospitalization. The $E$. coli JIE2288 isolate was also resistant to amikacin, gentamicin, tobramycin, amoxicillin/clavulanic acid, cefotaxime, ceftazidime, cefepime, cefoxitin, trimethoprim/sulfamethoxazole, and ciprofloxacin, according to EUCAST breakpoints. The isolate was susceptible to piperacillin/tazobactam (MIC of $<4 / 4 \mu \mathrm{g} / \mathrm{mL}$ ) and meropenem (MIC of $<1 \mu \mathrm{g} / \mathrm{mL}$ ). The patient had no history of overseas travel in the previous 5 years, and while she was in the ICU, no other ICU patients had a recognized colistin-resistant infection and none were receiving colistin treatment. The patient was housed in a single room, and standard infection-control precautions (disposable gown and gloves) were used. The patient was treated with a course of meropenem, and subsequent cultures of her urine did not contain colistin-resistant $E$. coli.

In July 2013, a second colistin-resistant $E$. coli strain (herein designated JIE3685; colistin MIC of $>4 \mu \mathrm{g} / \mathrm{mL}$ ) was referred to our laboratory after being isolated from the urine of a 71-year-old woman with diabetes mellitus. The woman had sought care from a community physician in Newcastle, New South Wales, for a urinary tract infection. She had had no healthcare contact in the previous 2 years and no history of travel outside Australia in the previous 5 years. JIE3685 was also resistant to cefotaxime, cefepime, ciprofloxacin, gentamicin, tobramycin, and trimethoprim/ 
sulfamethoxazole, but it was susceptible to amoxicillin/ clavulanic acid, ceftazidime, piperacillin/tazobactam, meropenem, and amikacin.

We obtained colistin-resistant transconjugants from E. coli JIE2288 and JIE3685 essentially as previously described (4) and, using PCR, confirmed that they carried $m c r-1$. We subjected DNA prepared from the original JIE2288 and JIE3685 isolates and transconjugants to paired-end sequencing (NextSeq 500 platform; Illumina, San Diego, CA, USA) and assembled the sequences by using SPAdes 3.7.1 (http://bioinf.spbau.ru/ spades). Using PlasmidFinder (https://cge.cbs.dtu.dk/ services/PlasmidFinder/), we identified IncI2 replicons in both isolates, and using the MLST tool on the Centre for Genomic Epidemiology website (https://cge.cbs.dtu. $\mathrm{dk} /$ services/MLST/), we identified JIE2288 as sequence type (ST) 167 (clonal complex [CC] 10) and JIE3865 as ST93 (CC168). Other ST167 isolates carrying $m c r-1$ have been reported; for example, $m c r-1$ was carried on a $\approx 65-\mathrm{kb}$ IncI2 plasmid and the bla $_{\mathrm{NDM}-9}$ gene on a different plasmid in an isolate from chicken meat in China in 2014 (5). Furthermore, ST93 (CC168) is a known zoonotic pathogenic strain previously associated with transmission of $m c r-1$ from animals to humans in Laos (6) and China (7).

Most of the IncI2 plasmid from E. coli JIE2288 that carries $m c r-1$ (designated pJIE2288-1) was assembled as a single contig, missing only the shufflon region that rearranges to change the end of the pilV gene (8), causing assembly issues that may result in apparent differences between plasmids carrying mcr-1 (9). We obtained the shufflon segments of pJIE2288-1 by mapping raw reads to individual shufflon segments from the archetypal IncI2 plasmid R721
(GenBank accession no. AP002527) and then assembled them in the same order as in R721 to generate a complete plasmid sequence (8). An additional plasmid, carrying the $b l a_{\text {CTX-M-14a }}$ gene (IncI1, designated pJIE2288-2), was identified in JIE2288. BLAST (https://blast.ncbi.nlm.nih.gov/ Blast.cgi) searches with 2 contigs from JIE3685 identified several closely matching plasmids (Table). We used pEC51 , which had been sequenced by long-read methods, as a reference to resolve a repeated region and as a template for the shufflon, thus enabling complete assembly of a plasmid designated pJIE3685-1. We submitted the sequences of pJIE2288-1 and pJIE3685-1 to GenBank under accession numbers KY795977 and KY795978, respectively.

pJIE2288-1 and pJIE3685-1 each carry mcr-1 as the only resistance gene (located in the same position), and both lack ISApl1, but they do have differences in the remainder of their IncI2 backbones. pJIE2288-1 (60.733 kb) is closely related to 9 other sequenced $m c r-1$ plasmids (differing by only a few single nucleotide changes in backbone regions), some of which carry ISApl1 upstream of $m c r-1$, another IS, or both (Table). pJIE3685-1 (60.960 kb) is closely related to 6 other IncI $2 \mathrm{mcr}-1$ plasmids (again, differing by only a few single nucleotide changes), all of which also lack ISApl1 (Table).

We noted differences between pJIE2288-1 and pJIE3685-1 in the $m c r-1$ promoter (11) and putative ribosome binding site and these or other differences in this region in available $m c r-1$ sequences (Figure). We examined the chromosomal arnBCADTEF, pmrAB, and phoPQ genes of each isolate but did not identify any of the changes previously reported in association with colistin resistance in E. coli (12). This finding suggests that $m c r-1$ may be the sole phenotype determinant in these 2 strains.

\begin{tabular}{|c|c|c|c|c|c|c|}
\hline $\begin{array}{l}\text { Study plasmid, } \\
\text { related plasmids }\end{array}$ & $\begin{array}{c}\text { GenBank } \\
\text { accession no. }\end{array}$ & Genus and species & Source & Country & Year & $\begin{array}{l}\text { Insertion } \\
\text { sequence }\end{array}$ \\
\hline pJIE2288-1 & KY795977 & E. coli & Human urine & Australia & 2011 & - \\
\hline pEG430-1 & LT174530 & Shigella sonnei & Human feces & Vietnam & 2008 & Apl1 \\
\hline pHSSH22-MCR1 & KX856067 & Salmonella Typhimurium & Human feces & China & 2012 & \\
\hline pHSSH23-MCR1 & KX856068 & Salmonella Enteritidis & Human feces & China & 2012 & Apl1 \\
\hline pHNSHP45 & KP347127 & E. coli & Pig farm & China & 2013 & Apl1, 683 \\
\hline pHeNE867† & KU934208 & E. coli & Chicken & China & 2013 & - \\
\hline pABC149-MCR-1 & KX013538 & E. coli & Human blood & UAE & 2013 & Apl1 \\
\hline pEc_04HAE 12 & KX592672 & E. coli & Human blood & China & 2014 & 1 \\
\hline pR150626 & KY120366 & Salmonella Typhimurium & Human & Taiwan & & - \\
\hline pEC006 & KY471144 & E. coli & & South Koreaf & & 150 \\
\hline pJIE3685-1 & KY795978 & E. coli & Human urine & Australia & 2011 & - \\
\hline pEC5-1 & CP016185 & E. coli & Chicken liver & Malaysia & 2013 & 1 \\
\hline $\mathrm{pEC} 13-1$ & СР016186 & E. coli & Pond water & Malaysia & 2013 & - \\
\hline pS2.14-2 & СР016187 & E. coli & Chicken feed & Malaysia & 2013 & - \\
\hline pEc 20COE13§ & KY012274 & E. coli & Human blood & China & 2014 & - \\
\hline pBA77-MCR-1 & KX013539 & E. coli & Human urine & Bahrain & 2015 & 1294 \\
\hline $\mathrm{pP} 111$ & KY120365 & Salmonella Typhimurium & Pig & Taiwan & & - \\
\hline
\end{tabular}




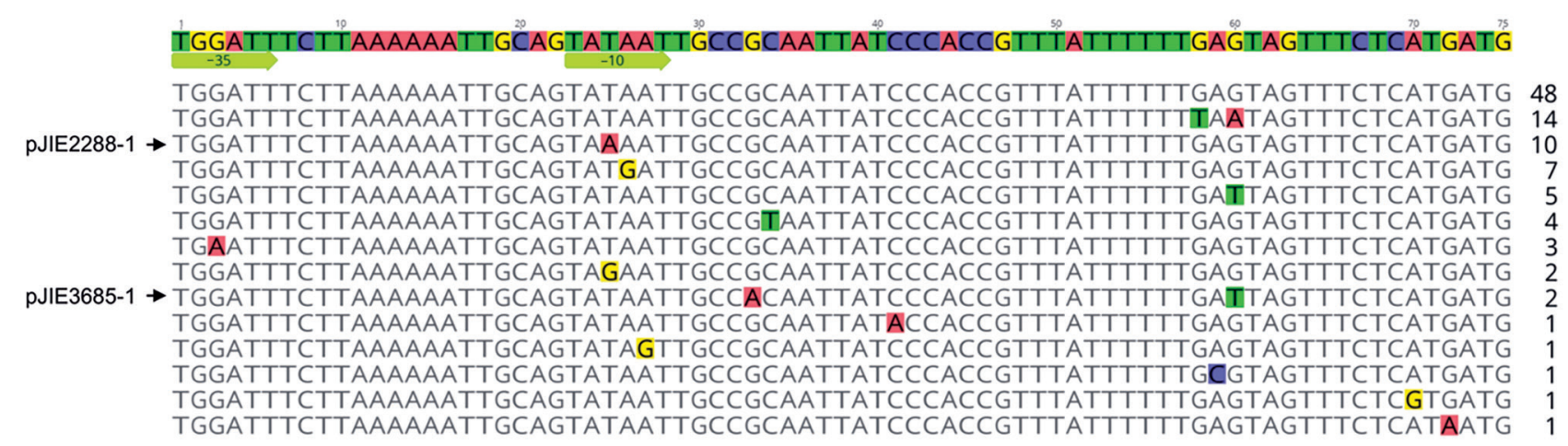

Figure. Differences in promoter and ribosome binding site regions of $m c r-1$ in plasmids from Escherichia coli in Australia (indicated by arrows) and in other sequences available from GenBank. The sequences end with the ATG start codon of $m c r-1$ and a second ATG codon that follows it. The -35 and -10 regions of the proposed promoter (11) are indicated by arrows. The numbers to the right indicate how many times each variant has been seen among available sequences.

\section{Conclusions}

We describe 2 E. coli isolates collected from patients in different cities in Australia $\approx 2$ years apart that belong to STs previously associated with $m c r-1$ and which carry this gene on plasmids that are virtually identical to previously described $m c r-1$-bearing plasmids from Asia and the Middle East. Multiple importations of resistant isolates into the community microflora are to be expected, and these 2 case-patients in Australia had no history of travel outside Australia or direct links to each other. Australia's regional neighbors use colistin extensively in agriculture (13), but in Australia, its usage in agriculture and healthcare is minimal, and neither colistin nor polymyxin drugs appear in the top 20 antimicrobial drugs prescribed in Australia (14). Notwithstanding the limitations of this opportunistic study, our findings indicate that colistin resistance is unusual among Enterobacteriaceae in Australia and that $m c r-1$ is neither a key mechanism nor yet widely disseminated in this country despite multiple importation events.

\section{Acknowledgment}

We thank Alberto Quesada Molina for providing the control isolate for $m c r-1$ testing.

A.N.G and J.R.I. were supported in part by the National Health and Medical Research Council of Australia (grants G1046886 and PF1002076, respectively).

Mr. Ellem is a hospital scientist in the General Microbiology Unit of the Centre for Infectious Diseases and Microbiology Laboratory Services, Westmead Hospital, New South Wales Health Pathology. His research interests are diagnostic methods for rapidly identifying bacterial pathogens and antibiotic resistance in gram-negative bacteria.

\section{References}

1. Liu YY, Wang Y, Walsh TR, Yi LX, Zhang R, Spencer J, et al. Emergence of plasmid-mediated colistin resistance mechanism MCR-1 in animals and human beings in China: a microbiological and molecular biological study. Lancet Infect Dis. 2016;16:161-8. http://dx.doi.org/10.1016/S1473-3099(15)00424-7

2. Snesrud E, He S, Chandler M, Dekker JP, Hickman AB, McGann P, et al. A model for transposition of the colistin resistance gene $m c r-1$ by ISApl1. Antimicrob Agents Chemother. 2016;60:6973-6. http://dx.doi.org/10.1128/AAC.01457-16

3. Schwarz S, Johnson AP. Transferable resistance to colistin: a new but old threat. J Antimicrob Chemother. 2016;71:2066-70. http://dx.doi.org/10.1093/jac/dkw274

4. Valenzuela JK, Thomas L, Partridge SR, van der Reijden T, Dijkshoorn L, Iredell J. Horizontal gene transfer in a polyclonal outbreak of carbapenem-resistant Acinetobacter baumannii. J Clin Microbiol. 2007;45:453-60. http://dx.doi.org/10.1128/JCM.01971-06

5. Yao X, Doi Y, Zeng L, Lv L, Liu JH. Carbapenem-resistant and colistin-resistant Escherichia coli co-producing NDM-9 and MCR-1. Lancet Infect Dis. 2016;16:288-9. http://dx.doi.org/ 10.1016/S1473-3099(16)00057-8

6. Olaitan AO, Thongmalayvong B, Akkhavong K, Somphavong S, Paboriboune P, Khounsy S, et al. Clonal transmission of a colistin-resistant Escherichia coli from a domesticated pig to a human in Laos. J Antimicrob Chemother. 2015;70:3402-4.

7. Zhang XF, Doi Y, Huang X, Li HY, Zhong LL, Zeng KJ, et al. Possible transmission of $m c r-1$-harboring Escherichia coli between companion animals and human. Emerg Infect Dis. 2016;22:167981. http://dx.doi.org/10.3201/eid2209.160464

8. Brouwer MS, Tagg KA, Mevius DJ, Iredell JR, Bossers A, Smith HE, et al. IncI shufflons: assembly issues in the next-generation sequencing era. Plasmid. 2015;80:111-7. http://dx.doi.org/10.1016/ j.plasmid.2015.04.009

9. Suzuki S, Ohnishi M, Kawanishi M, Akiba M, Kuroda M. Investigation of a plasmid genome database for colistin-resistance gene $\mathbf{m c r}$-1. Lancet Infect Dis. 2016;16:284-5. http://dx.doi.org/ 10.1016/S1473-3099(16)00008-6

10. Yang YQ, Li YX, Song T, Yang YX, Jiang W, Zhang AY, et al. Colistin resistance gene $m c r-1$ and its variant in Escherichia coli isolates from chickens in China. Antimicrob Agents Chemother. 2017:16:e1201-61. http://dx.doi.org/ 10.1128/ AAC.01204-16

11. Poirel L, Kieffer N, Brink A, Coetze J, Jayol A, Nordmann P. Genetic features of MCR-1-producing colistin-resistant Escherichia coli isolates in South Africa. Antimicrob Agents Chemother. 2016;60:4394-7. http://dx.doi.org/10.1128/AAC.00444-16 
12. Olaitan AO, Morand S, Rolain JM. Mechanisms of polymyxin resistance: acquired and intrinsic resistance in bacteria. Front Microbiol. 2014;5:643. http://dx.doi.org/10.3389/fmicb.2014.00643

13. Nguyen NT, Nguyen HM, Nguyen CV, Nguyen TV, Nguyen MT, Thai HQ, et al. Use of colistin and other critical antimicrobials on pig and chicken farms in southern Vietnam and its association with resistance in commensal Escherichia coli bacteria. Appl Environ Microbiol. 2016;82:3727-35. http://dx.doi.org/10.1128/ AEM.00337-16
14. Australian Commission on Safety and Quality in Health Care. AURA 2016: first Australian report on antimicrobial use and resistance in human health. Sydney: the Commission; 2016.

Address for correspondence: Jonathan R. Iredell, CIDM, Level 3, ICPMR Bldg, Westmead Hospital, Westmead, NSW 2145, Australia; email: jonathan.iredell@sydney.edu.au

\section{November 2016: Bacterial Pathogens}

- Transmission of Babesia microti Parasites by Solid Organ Transplantation

- Immune Responses to Invasive Group B Streptococcal Disease in Adults

- Ambulatory Pediatric Surveillance of Hand, Foot and Mouth Disease As Signal of an Outbreak of Coxsackievirus A6 Infections, France, 2014-2015

- Increased Hospitalization for Neuropathies as Indicators of Zika Virus Infection, according to Health Information System Data, Brazil

- Global Escherichia coli Sequence Type 131 Clade with bla ${ }_{\mathrm{CTX}-\mathrm{M}-27}$ Gene
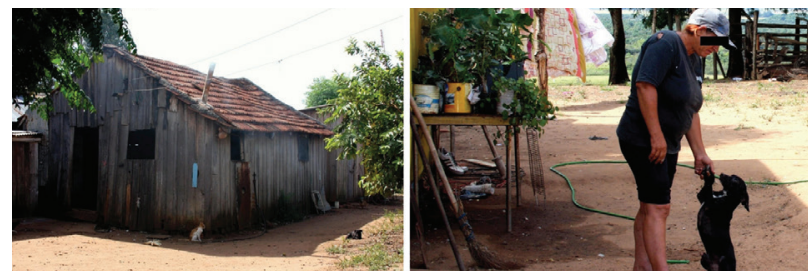

- Multidrug-Resistant Corynebacterium striatum Associated with Increased Use of Parenteral Antimicrobial Drugs

- Risk Factors for Middle East Respiratory Syndrome Coronavirus Infection among Healthcare Personnel

- Epidemiology of La Crosse Virus Emergence, Appalachian Region, United States

- Reassortant Eurasian Avian-Like Influenza $\mathrm{A}(\mathrm{H} 1 \mathrm{~N} 1)$ Virus from a Severely III Child, Hunan Province, China, 2015

- Serotype IV Sequence Type 468 Group B Streptococcus Neonatal Invasive Disease, Minnesota, USA

- Capsular Switching and Other Large-Scale Recombination Events in Invasive Sequence Type 1 Group B Streptococcus

- Changing Pattern of Chlamydia trachomatis Strains in Lymphogranuloma Venereum Outbreak, France, 2010-2015
- ESBL-Producing and MacrolideResistant Shigella sonnei Infections among Men Who Have Sex with Men, England, 2015

- Early Growth and Neurologic Outcomes of Infants with Probable Congenital Zika Virus Syndrome

- Severe Fever with Thrombocytopenia Syndrome Complicated by Co-infection with Spotted Fever Group Rickettsiae, China

- Guinea Worm (Dracunculus medinensis) Infection in a Wild-Caught Frog, Chad

- Dog-Mediated Human Rabies Death, Haiti, 2016

- Staphylococcus aureus Colonization and Long-Term Risk for Death, United States

- Group B Streptococcus Serotype III Sequence Type 283 Bacteremia Associated with Consumption of Raw Fish, Singapore

- Group B Streptococcus Sequence Type 283 Disease Linked to Consumption of Raw Fish, Singapore

- Novel Levofloxacin-Resistant Multidrug-Resistant Streptococcus pneumoniae Serotype 11 A Isolate, South Korea

- Imported Chikungunya Virus Strains, Taiwan, 2006-2014

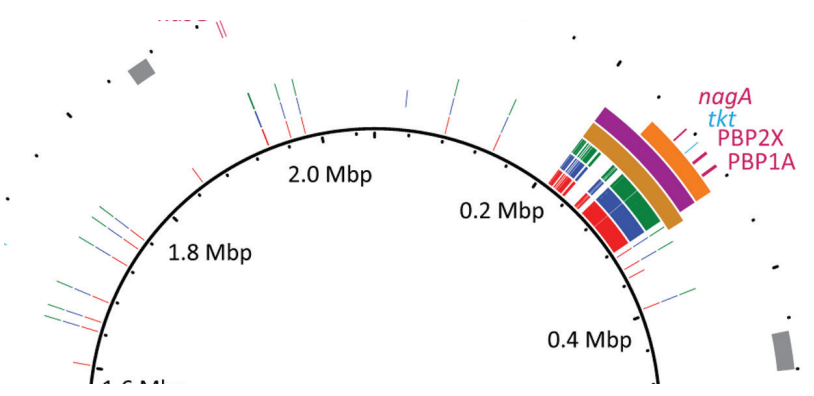

